\title{
Performance Evaluation of an Optoelectronic Oscillator Based on a Band-pass Microwave Photonic Filter Architecture
}

\author{
Ana Gabriela CORREA-MENA ${ }^{1,2}$, Ignacio Enrique ZALDÍVAR-HUERTA ${ }^{1}$, \\ Min Won LEE ${ }^{3}$, Alejandro GARCÍA-JUÁREZ ${ }^{4}$ Luis A. GARCÍA-DELGADO \\ ${ }^{1}$ Dept. de Electrónica, Instituto Nacional de Astrofísica, Óptica y Electrónica, \\ Apartado Postal 51 y 216, 72000, Puebla, México \\ ${ }^{2}$ Dept. de Ciencias de la Computación y Electrónica, Universidad Técnica Particular de Loja, \\ San Cayetano Alto, 1101608, Loja, Ecuador \\ ${ }^{3}$ Laboratoire de Physique des Lasers (UMR CNRS 7538), Université Paris 13, Sorbonne Paris Cité, \\ 93430, Villetaneuse, France \\ ${ }^{4}$ Dept. de Investigación en Física, Universidad de Sonora, Blvd. Luis Encinas y Rosales S/N, 83000, Hermosillo, México \\ \{agcorrea, zaldivar\}@inaoep.mx
}

Submitted February 24, 2017 / Accepted April 19, 2017

\begin{abstract}
The experimental performance evaluation of an optoelectronic oscillator based on a band-pass microwave photonic filter architecture is carried out. The novelty of this proposal resides in the fact that the architecture used allows enhancing the free spectral range of the optoelectronic oscillator. Considering the optical spectral characteristics of the multimode laser diode used as an optical source, the length and the chromatic dispersion parameter of the optical fiber which acts as a feedback loop, it is possible to determine the appearance of a series of spectrally pure microwave signals widely spaced. In particular, the experimental results show a phase noise as low as $-92.69 \mathrm{dBc} / \mathrm{Hz}$ at $10 \mathrm{kHz}$ offset frequency from the $2.26 \mathrm{GHz}$ carrier for an optical delay line of $25.24 \mathrm{~km}$ and a $Q$ factor of $2.04 \times 10^{9}$.
\end{abstract}

\section{Keywords}

Optoelectronic oscillator, band-pass microwave photonic filter, microwave signals, phase noise

\section{Introduction}

Since Optoelectronic Oscillators (OEOs) were proposed by Yao and Maleki [1] in 1996, these systems have been attracting a lot of interest for their capabilities in generating high frequency low phase-noise microwave signals. Thanks to these advantages, currently OEOs find applications in optical and wireless communication, radar, signal processing, sensors, metrology and radio astronomy [2], [3]. The simplest scheme of an OEO is composed by a pump laser (usually a Distributed Feedback Laser DFB), and a feedback loop that includes an intensity modulator, an optical fiber delay line, a fast photo-detector, a RF
Band-Pass Filter (BPF), and a RF amplifier [4-7]. Basically, the principle of operation of an OEO is based on the energy stored on the optical fiber delay line, which is usually from a few meters to tens of kilometers, ensuring in this way several oscillation modes. The selection of a particular single oscillation mode can be obtained by using: delay line OEO [8], a dual-loop OEO [9], a dual injectionlocked OEO [5], or a fiber ring resonator [10]. Due to the modes are closely spaced, the aforementioned configurations require the use of an ultra-narrow BPF for the selection of a single mode injecting into the feedback loop. However, the use of BPFs results in a lower RF stability and higher phase noise [11]. In this regard, some works have reported techniques that avoid the use of any electric microwave filter. For example, in [12], a novel optically tunable OEO scheme by using a tunable laser and an injection locking of a Fabry-Perot-Laser Diode is described; in [11], the use of a Fabry-Perot etalon optical filter as the mode selector instead of an RF filter is experimentally demonstrated.

On the other hand, Microwave Photonic Filters (MPFs) have several applications to microwave and optical systems [13]. About this theme, in the past we have proposed a novel band-pass MPF topology whose frequency response in the range of $0.01-10 \mathrm{GHz}$ comprises a series of band-pass windows centered at frequencies that can be tailored to the function of the intermodal separation that characterizes a Multimode Laser Diode (MLD), the length and chromatic dispersion parameter of the optical fiber used [14].

Taking into account the precedent paragraphs, the aim of this work is to undertake the experimental performance evaluation of an optoelectronic oscillator based on the band-pass microwave photonic filter architecture reported in [14]. The novelty of this proposal resides in the fact that 
the architecture used allows enhancing the Free Spectral Range (FSR) of the optoelectronic oscillator. Thus, our hypothesis resides in demonstrating that the chromatic dispersion parameter of the optical fiber delay line, as well as the spectral characteristics of the MLD can be used in advantageous manner to obtain spectrally pure microwave signals widely spaced (in the order of $\mathrm{GHz}$ ). Therefore, it is not necessary in our configuration to employ any electrical BPF in the feedback loop. In particular, it is demonstrated, experimentally, that using an optical delay line of $25.24 \mathrm{~km}$, a $Q$ factor of $2.04 \times 10^{9}$ and a phase noise as low as $-92.69 \mathrm{dBc} / \mathrm{Hz}$ at $10 \mathrm{kHz}$ offset frequency are obtained from the $2.26 \mathrm{GHz}$ carrier.

This paper is organized as follows. Section 2 provides the basic theory of the OEO operation principle. Experimental procedures and results are described in Sec. 3. Finally, the main conclusions are included in Sec. 4.

\section{Basic Theory}

Figure 1 depicts a conventional OEO scheme which normally consists of a pump laser (DFB), followed by a Mach-Zehnder Intensity Modulator (MZ-IM). The light from the output of the MZ-IM is injected into an optical fiber delay line, and detected by the fast Photo-Detector (PD). An electrical amplifier and a BPF are used to provide sufficient gain to the loop and to select the desired oscillation frequency, respectively. Finally, the output of the BPF is connected to the electric-input port of the modulator to complete the feedback loop, allowing in this way the oscillation of the system [1], [2].

The Quality factor $\left(Q_{\mathrm{OEO}}\right)$ parameter is defined as the ratio between the oscillation frequency $\left(f_{\text {osc }}\right)$ and the full width at half-maximum $\left(\Delta f_{\mathrm{FWHM}}\right)$ of the generated signal, and it is [1]

$$
Q_{\mathrm{OEO}}=\frac{f_{\mathrm{osc}}}{\Delta f_{\mathrm{FWHM}}} .
$$

The denominator in (1) is given as [1]

$$
\Delta f_{\mathrm{FWHM}}=\frac{1}{2 \pi} \frac{\delta}{\tau^{2}}
$$

where $\tau$ is the total group delay of the loop given by $\tau=L n / c$, where $L$ and $n$ are the length and refractive index of the optical fiber used as optical delay line, respectively, and $c$ is the speed of light in free space. The input-noise-tosignal-ratio $(\delta)$ of the oscillator is a function of the input noise density $\left(\rho_{\mathrm{N}}\right)$, the total oscillating power $\left(P_{\mathrm{osc}}\right)$, and the gain of the electrical amplifier $\left(G_{\mathrm{A}}\right)$, that is [15]

$$
\delta=\frac{\rho_{\mathrm{N}} G_{\mathrm{A}}^{2}}{P_{\mathrm{osc}}} .
$$

The power spectral density $\left(S_{\mathrm{RF}}\right)$ of the OEO for a frequency offset $f^{\prime}$ is defined as [15]

$$
S_{\mathrm{RF}}\left(f^{\prime}\right)=\delta /\left[\left(\frac{\delta}{2 \tau}\right)^{2}+(2 \pi)^{2}\left(\pi f^{\prime}\right)^{2}\right]
$$

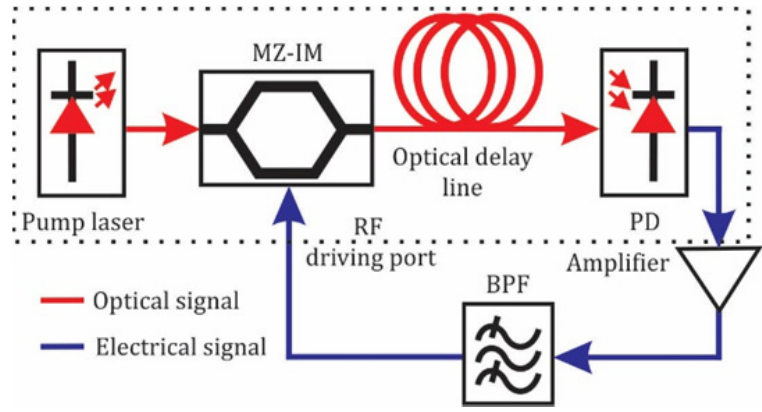

Fig. 1. Basic architecture of an optoelectronic oscillator.

This last relationship is used to compute the phase noise, given as [1]

$$
\text { phase noise }=10 \log \left(\frac{S_{\mathrm{RF}}\left(f^{\prime}\right)}{10^{-3}}\right)-20 \log \left(f^{\prime}\right) .
$$

The phase noise is determined as a single value measured at a frequency offset from the carrier [16], and it is notoriously independent of the oscillation frequency.

On the other hand, it must be noted that the structure enclosed within the black dotted box of Fig. 1 corresponds to the MPF architecture reported in [14]. In this reference, a detailed mathematical analysis was carried out to demonstrate that, when a MLD is used and a continuous RF signal supplied by an electrical signal generator is applied to the electric port of the MZ-IM, the filtering of microwave signals is accomplished. Therefore, the central frequency $f_{n}$ for these filtered signals is a function of the length of the optical fiber $L$, the chromatic fiber-dispersion parameter $D$ associated to the optical fiber, the intermodal separation $\delta \lambda$ of the MLD, and its value can be computed as [14]

$$
f_{n}=\frac{n}{D L \delta \lambda}
$$

where $n$ is a positive integer $(n=1,2, \ldots)$.

Referring again to the scheme depicted in Fig. 1 and considering a MLD as the pump laser (instead of a DFB), the continuous RF signal issued by the PD is amplified, filtered and connected to the electric port of the MZ-IM forming a feedback loop. At this point, two effects are manifested: (i) the filtering effect is brought into play, and (ii) the MPF system operates as an oscillator. Thus, the central frequency of each MPF filtered signal corresponds to the oscillation frequency $f_{\mathrm{OEO}}$ of the OEO, that is $f_{n}=f_{\text {OEO }}$. Now, $n$ of (6) is the $n^{\text {th }}$ mode of the oscillation frequency.

\section{Proposed OEO and Experimental Results}

The schematic diagram of the proposed OEO using a MLD as pump laser is shown in Fig. 2. The block enclosed into the dotted box is the MPF, whose frequency response exhibits a series of filtered microwave band-pass windows characterized by a bandwidth at $-3 \mathrm{~dB}$ around of 


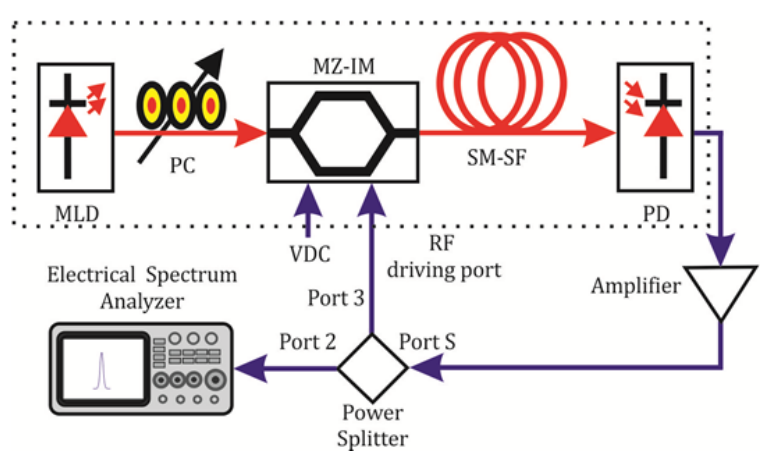

Fig. 2. Proposed OEO using a band-pass microwave photonic filter architecture.

$300 \mathrm{MHz}$. In contrast to Fig. 1, the absence of a BPF in the feedback loop is notorious. In the following, we describe in detail the operation of the proposed OEO architecture.

In a first step, the MLD used in this experiment is optically characterized by means of an optical spectrum analyzer (Agilent, model 86143B). The MLD is operated by a temperature-controller, guaranteeing in this way stability to thermal fluctuations and a low value of Relative Intensity Noise (RIN) [17]. Figure 3 illustrates the emission spectrum of the MLD for a current of $20 \mathrm{~mA}$, obtaining the optical parameters: $\lambda_{0}=1544.25 \mathrm{~nm}$ and $\delta \lambda=1.1 \mathrm{~nm}$. The optical central wavelength value guarantees that the MZIM operates in the appropriate optical bandwidth.

The light issued by the MLD is injected to the MZ-IM (Photline, MXAN-LN-20-bandwith-20 GHz, insertion loss of $2.7 \mathrm{~dB}, \mathrm{~V}_{\pi}=3.0 \mathrm{~V}$, operating wavelength from 1530 to $1580 \mathrm{~nm}$ ) through a Polarization Controller (PC) in order to optimize the polarization of the MLD output. The modulated light is launched to a spool of Single-Mode Standard Fiber (SM-SF, Corning SM-SF-28, $\alpha=0.22 \mathrm{~dB} / \mathrm{km}, D=$ $15.81 \mathrm{ps} / \mathrm{nm}-\mathrm{km} @ 1550 \mathrm{~nm}$ ) of $25.24 \mathrm{~km}$ (available in our laboratory). The light coming from the SM-SF is converted to its corresponding electrical signal by a fast PD (Miteq, model DR-125G-A, bandwidth-13 GHz, and $\mathfrak{R}=0.9 \mathrm{~A} / \mathrm{W}$ ). An electrical amplifier is used to provide sufficient gain (approximately $22 \mathrm{~dB}$ ). The amplified electrical signal is separated by means of a power splitter. Port 3 is fed to the RF port of the MZ-IM in order to close the OEO feedback loop, whereas output of Port 2 is connected to an Electrical Spectrum Analyzer (ESA) to evaluate the frequency response of the OEO.

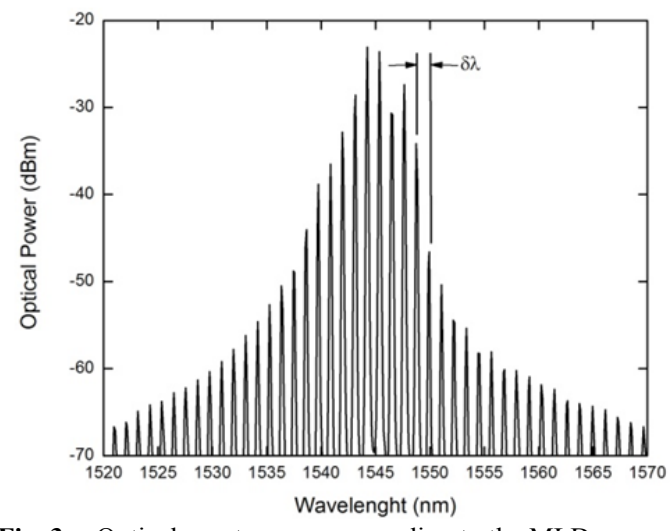

Fig. 3. Optical spectrum corresponding to the MLD.

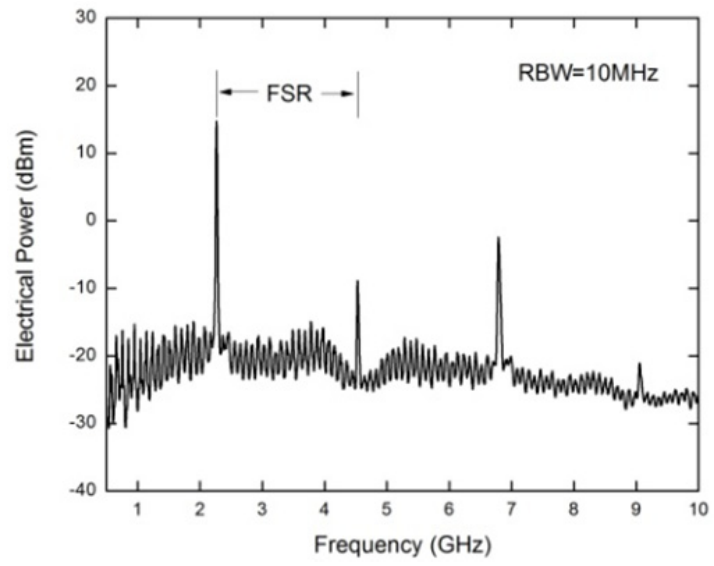

Fig. 4. Measured frequency response of the OEO using an MLD as pump laser.

\begin{tabular}{|c|c|c|c|}
\hline $\begin{array}{c}\text { Oscillation } \\
\text { Frequency }\end{array}$ & $\begin{array}{c}\text { Experimental } \\
{[\mathrm{GHz}]}\end{array}$ & $\begin{array}{c}\text { Theoretical } \\
{[\mathrm{GHz}]}\end{array}$ & $\begin{array}{c}\text { Error } \\
{[\%]}\end{array}$ \\
\hline$f_{1}$ & 2.26 & 2.27 & 0.44 \\
\hline$f_{2}$ & 4.53 & 4.55 & 0.43 \\
\hline$f_{3}$ & 6.80 & 6.83 & 0.43 \\
\hline
\end{tabular}

Tab. 1. Experimental and theoretical oscillation frequency of the proposed OEO scheme.

Figure 4 depicts the OEO frequency response measured by the ESA (Anritsu, model MS2830A-044). The presence of a series of stable signals located at $f_{1}=2.26 \mathrm{GHz}(14.63 \mathrm{dBm}, S N R=31.63 \mathrm{~dB}), f_{2}=4.53 \mathrm{GHz}$ $(-9.25 \mathrm{dBm}, \quad S N R=7.75 \mathrm{~dB}), \quad$ and $\quad f_{3}=6.80 \mathrm{GHz}$ $(-3.93 \mathrm{dBm}, S N R=13.07 \mathrm{~dB})$ is clearly appreciable. These experimental results are in good agreement with the theoretical results obtained applying (6), that are: $f_{1}=2.27 \mathrm{GHz}, f_{2}=4.55 \mathrm{GHz}, f_{3}=6.83 \mathrm{GHz}$. Experimental and theoretical results are summarized in columns two and three of Tab. 1, respectively.

Due to the periodicity of the optical spectrum (see Fig. 3), the experimental microwave signals are perfectly spaced at $2.27 \mathrm{GHz}$, in contrast, the FSR reported in [8] is closely spaced at $150 \mathrm{kHz}$ (DFB as pump laser). In the latter, adding a BPF in the feedback loop of the OEO is necessary, while our system does not require. Given that the optical fiber, which plays the role of optical delay, is very sensitive to the surrounding environment, measurements were carried out in a climate-controlled room $\left(25^{\circ} \mathrm{C}\right)$ in order to guarantee accuracy and stability for the microwave signals.

In particular, the microwave signal centered at 2.26 GHz was selected in order to achieve the performance evaluation of this optoelectronic oscillator. The procedure of computation of $Q$ at the oscilation frequency of $2.26 \mathrm{GHz}$ is described below.

Initially, the input noise density is mesured experimentally determining $\rho_{\mathrm{N}}=1 \times 10^{-13} \mathrm{~W} / \mathrm{Hz}$. From Fig. 4, the total oscillating power is determined as $P_{\text {osc }}=0.029 \mathrm{~W}$. Knowing that the gain of the amplifier used is $22.45 \mathrm{~dB}$, then $G_{\mathrm{A}}=175.79$. Afterwards, considering $L=25.24 \mathrm{~km}$ and $n=1.47$ for the optical fiber, thus $\tau=124 \mu \mathrm{s}$. The knowledge of these parameters allows, in the first step, the 


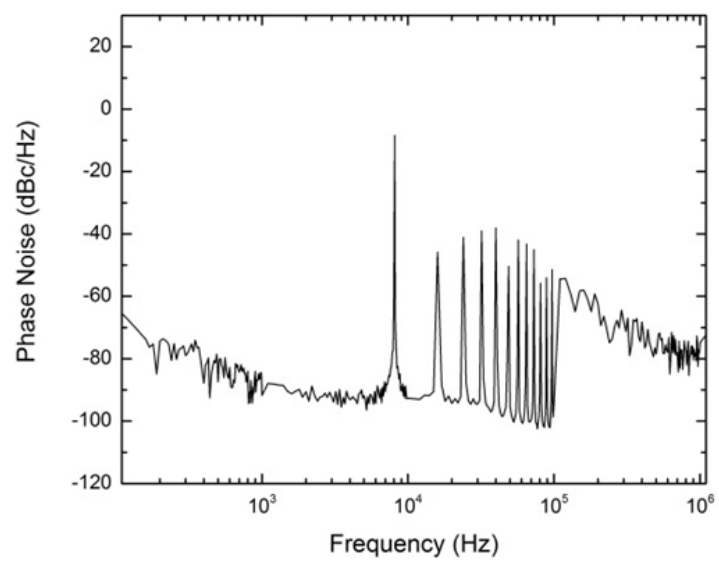

Fig. 5. Phase noise measurement.

\begin{tabular}{|c|c|c|}
\hline $\begin{array}{c}\text { Frequency } \\
\text { Offset }[\mathrm{Hz}]\end{array}$ & $\begin{array}{c}\text { Theoretical } \\
{[\mathrm{dBc} / \mathrm{Hz}]}\end{array}$ & $\begin{array}{c}\text { Experimental } \\
{[\mathrm{dBc} / \mathrm{Hz}]}\end{array}$ \\
\hline $10^{2}$ & -67.55 & -69.65 \\
\hline $10^{3}$ & -107.55 & -91.75 \\
\hline $10^{4}$ & -147.55 & -92.69 \\
\hline $10^{5}$ & -187.55 & -94.76 \\
\hline
\end{tabular}

Tab. 2. Phase noise measurement of the $\mathrm{OEO}$ at $2.26 \mathrm{GHz}$.

use of (3) to obtain the input-noise-to-signal-ratio of the oscillator $\left(\delta=1.065 \times 10^{-7} \mathrm{~Hz}^{-1}\right)$. This result is substituted in (2) to compute the full width at half-maximum of the generated signal $\left(\Delta f_{\mathrm{FWHM}}=1.10 \mathrm{~Hz}\right)$. Then, the quality factor $Q$ is determined by using (1), obtaining $Q_{\mathrm{OEO}}=$ $2.04 \times 10^{9}$ that is in good agreement with values reported in the literature $[5,15,18]$. Subsequently, the power spectral density and the phase noise is determined by (4) and (5), respectively.

Figure 5 depicts the phase noise measured by the ESA and the presences of spurious peaks, according to the literature, are attributed to the delay by the long optical fiber in the feedback loop [19]. Finally, the theoretical and experimental phase noise measurements are listed in Tab. 2 .

\section{Conclusions}

We have carried out the experimental performance evaluation of an optoelectronic oscillator based on a bandpass MPF architecture. The key novelty of the proposed OEO scheme is the advantageous use of the chromatic dispersion parameter of the optical fiber, as well as the optical spectral characteristics of the MLD to select a particular oscillation frequency. The band-pass MPF acts as an oscillation frequency selector. Thus, the oscillation frequency is linked to the band-pass frequency response of the MPF that allows a wide FSR without any additional electronic microwave filter. In particular, the microwave signal at $2.26 \mathrm{GHz}$ was analyzed, obtaining a high quality factor $\left(Q=2.04 \times 10^{9}\right)$ and a phase noise as low as $-92.69 \mathrm{dBc} / \mathrm{Hz}$ at $10 \mathrm{kHz}$ offset frequency. Improvement is still possible if an RF low noise amplifier is used. Even more, the simplicity of the proposed OEO resides in the fact that, in contrast to previous reported works, it is not necessary to use additional optical devices, e.g., optical sources, Erbium Doped Fiber Amplifiers (EDFAs), optical filters, among others. Note that knowing the intermodal separation of the MLD, the length of the optical fiber and its associated chromatic dispersion parameter, it is possible to determine the theoretical oscillation frequency by means of (6). The intermodal separation of the MLD is a fixed value and it is provided by the manufacturer, the chromatic dispersion parameter could be tailored by using Photonic Crystal Fibers (PCFs) [20], however, the simplest way to select the oscillation frequency is by choosing an appropriate length of optical fiber. For example, for lengths of 10 , 15 and $20 \mathrm{~km}$, the corresponding FSRs are: 5.73, 3.83 and $2.87 \mathrm{GHz}$, respectively. Currently, we are working on a self-sustaining architecture for an optical fiber communication system in order to justify the use of a long length optical fiber. Inclusively, the feedback loop in an OEO scheme allows generating its own RF source, providing privacy and security in communication systems.

\section{Acknowledgments}

One of the authors, A. G. Correa-Mena would like to thank the Mexican Consejo Nacional de Ciencia y Tecnología (CONACyT) for the student scholarship (number 335148).

\section{References}

[1] YAO, X. S., MALEKI, L. Optoelectronic microwave oscillator. Journal of the Optical Society of America B, 1996, vol. 13, no. 8, p. 1725-1735. DOI: 10.1364/JOSAB.13.001725

[2] MALEKI, L. Sources: The optoelectronic oscillator. Nature Photonics Technology Focus, 2011, vol. 5, no. 12, p. 728-730. DOI: 10.1038/nphoton.2011.293

[3] WANG, P., XIONG, J., ZHANG, T., CHEN, D., et al. Frequency tunable optoelectronic oscillator based on a directly modulated DFB semiconductor laser under optical injection. Optics Express, 2015, vol. 23, no. 16, p. 20450-20458. DOI: 10.1364/OE.23.020450

[4] JI, Y., YAO, X. S., MALEKI, L. Compact optoelectronic oscillator with ultralow phase noise performance. Electronics Letters, 1999, vol. 35, no. 18, p. 1554-1555. DOI: 10.1049/el:19991027

[5] ZHOU, W., BLASCHE, G. Injection-locked dual opto-electronic oscillator with ultra-low phase noise and ultra-low spurious level. IEEE Transactions on Microwave Theory and Techniques, 2005, vol. 53, no. 3, p. 929-933. DOI: 10.1109/TMTT.2004.842489

[6] VOLYANSKIY, K., CHEMBO, Y., LARGER, L., RUBIOLA, E. Contribution of laser frequency and power fluctuations to the microwave phase noise of optoelectronic oscillators. Journal of Lightwave Technology, 2010, vol. 28, no. 18, p. 2730-2735. DOI: 10.1109/JLT.2010.2064230

[7] CORREA-MENA, A. G., HERNANDEZ-NAVA, P., ZALDIVAR-HUERTA, I. E., SALAS-CARIDAD, A. D., et al. Generation and distribution of microwave signals by using optoelectronic oscillators. In Proceedings of the 58th IEEE International Midwest Symposium on Circuits and Systems (MWSCAS). Fort Collins (CO, USA), 2015. p. 1-3. DOI: 10.1109/MWSCAS.2015.7282073 
[8] ROMISCH, S., KITCHING, J., FERRÈ-PIKAL, E., HOLLBERG, L., et.al. Performance evaluation of an optoelectronic oscillator. IEEE Transactions on Ultrasonics, Ferroelectrics, and Frequency Control, 2000, vol. 47, no. 5, p. 1159-1165. DOI: $10.1109 / 58.869060$

[9] ELIYAHU, D., MALEKI, L. Low phase noise and spurious level in multi-loop opto-electronic oscillators. In Proceedings of the Frequency Control Symposium and PDA Exhibition Jointly with the 17th European Frequency and Time Forum. Tampa (FL, USA), 2003, p. 405-410. DOI: 10.1109/FREQ.2003.1275126

[10] SALEH, K., MERRER, P. H., LlOPIS, O., CIBIEL, G. Optoelectronic oscillator based on fiber ring resonator: overall system optimization and phase noise reduction. In Proceedings of the International Frequency Control Symposium (FCS). Baltimore (MD, USA), 2012. p. 1-6. DOI: 10.1109/FCS.2012.6243650

[11] OZDUR, I., AKBULUT, M., HOGHOOGHI, N., MANDRIDIS, et al. Optoelectronic loop design with 1000 finesse Fabry-Perot etalon. Optics Letters, 2010, vol. 35, no. 6, p. 799-801. DOI: 0.1364/OL.35.000799

[12] WANG, W., YU, J., WU, B., HAN, B., et al. Optoelectronic oscillator based on injection locking of Fabry-Perot laser diode. In Proceedings of the Asia Communications and Photonics Conference and Exhibition (ACP 2010). Shanghai (China), 2010, p. 19-20. DOI: 10.1109/ACP.2010.5682854

[13] CAPMANY, J., ORTEGA, B., PASTOR, D. A tutorial on microwave photonic filters. Journal of Lightwave Technology, 2006, vol. 24, no. 1, p. 201-229.

[14] ZALDIVAR-HUERTA, I. E., GARCÍA-JUÁREZ, A., HERNÁNDEZ-NAVA, P., RODRIGUEZ-ASOMOZA, J. Experimental transmission in a fiber radio scheme using a microwave photonic filter operating at 2.8 GHz. IEICE Electronics Express, 2013, vol. 10, no. 3, p. 20130028. DOI: 10.1587/elex.10.20130028

[15] OKUSAGA, O., ZHOU, W., LEVY, E., et al. Experimental and simulation study of dual injection-locked OEOs. In Proceedings of the IEEE International Frequency Control Symposium, 2009 Joint with the 22nd European Frequency and Time Forum. Besancon (France), 2009, p. 875-879. DOI: 10.1109/FREQ.2009.5168312

[16] DEVGAN, P. A review of optoelectronic oscillators for high speed signal processing and applications. ISRN Electronics, 2013, article ID 401969, 16 p. DOI: 10.1155/2013/401969

[17] PASCHOTTA, R. Noise in laser technology. Optik \& Photonik, 2009, vol. 4, no. 2 , p. $48-50$.

[18] SALEH, K., BOUCHIER, A., MERRER, P. H., LLOPIS, O., et al. Fiber ring resonator based opto-electronic oscillator: phase noise optimization and thermal stability study. In Proceedings of the SPIE 7936, RF and Millimeter-Wave Photonics, 2011, 10 p. DOI: $10.1117 / 12.873755$

[19] NGUIMDO, R. M., SALEH, K., LIN, G., MATINENGHI, R., et al. Advances in optoelectronic oscillators. In Proceedings of the SPIE 9747, Terahertz, RF, Millimeter, and Submillimeter-Wave Technology and Applications IX, 2016, 5 p. DOI: $10.1117 / 12.2212037$

[20] EBNALI-HEIDARI, M., DEHGHAN, F., SAGHAEI, H., KOOHIKAMALI, F., et al. Dispersion engineering of photonic crystal fibers by means of fluidic infiltration. Journal of Modern Optics, 2012, vol. 59, no. 16, p. 1384-1390. DOI: $10.1080 / 09500340.2012 .715690$

\section{About the Authors...}

Ana Gabriela CORREA-MENA received her BS degree in Electronic and Telecommunications Engineering from Universidad Técnica Particular de Loja (Ecuador) in 2008 and M.S. degree in Electrical Engineering from Pontifícia Universidade Católica de Rio de Janeiro (Brazil) in 2013. She is currently a PhD student in Electronics from the Instituto Nacional de Astrofísica, Óptica y Electrónica (INAOE) (México). Her areas of research are optical fiber communication systems, microwave photonics. She is an IEEE member.

Ignacio Enrique ZALDIVAR-HUERTA received his BS degree in Electronic Engineering from Universidad Autónoma de Puebla (México) in 1992, his M.S degree in Microelectronics from INAOE (México) in 1995, and his PhD degree in sciences for engineering from Université de Franche-Comté (France) in 2001. Since 2002, he has been with the Department of Electronics of the INAOE. His research interests are optical fiber communication systems and electro-optic devices. He is a Senior Member of the IEEE and Member SPIE.

Min Won LEE received the MSc degree from Ajou University, Suwon, South Korea in 1998 and the PhD degree from the Université de Franche-Comté, Besançon, France in 2002. His doctoral research was concerned with chaotic dynamics behavior, chaos synchronization, optical telecommunications and chaos encryption. Since September 2011, he works as a lecturer in the Laboratoire de Physique des Lasers at the University of Paris 13. He works on semiconductor laser dynamics, nonlinear optics in nonlinear photonic crystals and electrical pulse-pumped OLED.

Alejandro GARCÍA-JUÁREZ received his BS degree in Electronics Engineering from Universidad Autónoma de Puebla, México in 1998, and his MS and PhD degrees in Optics with a specialty in optoelectronic systems from INAOE (México) in 1999 and 2005, respectively. Currently, he is a full-time professor-researcher in the Physics Department of Universidad de Sonora, México. His current research interests are primarily in optical fiber communication systems, microwave photonics and hybrid wireless visible light communication systems.

Luis GARCÍA-DELGADO received the B.S. degree in Electronic Engineering in 2006, M.Sc. and Ph.D. in Electric Engineering-Mechatronic and Control, in 2008 and 2012, respectively, all from Instituto Tecnológico de la Laguna, in Torreón, Mexico. Currently, he is a full-time professor-researcher in the Physics Department of Universidad de Sonora, México. 